\title{
Autopsy: The Dying Diagnostic
}

Angus Turnbull ${ }^{*}$ and Michael Osborn ${ }^{2}$

${ }^{1}$ Imperial College School of Medicine, Sir Alexander Fleming Building, South Kensington Campus, London, UK

${ }^{2}$ Department of Cellular Pathology, Imperial College NHS Healthcare Trust, London, UK

"Corresponding author: Angus Turnbull, Imperial College School of Medicine, Sir Alexander Fleming Building, South Kensington Campus, London, SW7 2AZ, UK, Tel: 44735650914; E-mail: ajt108@imperial.ac.uk

Rec Date: May 05, 2016; Acc Date: Jul 27, 2016; Pub Date: Aug 03, 2016

Copyright: ( 2016 Turnbull A, et al. This is an open-access article distributed under the terms of the Creative Commons Attribution License, which permits unrestricted use, distribution, and reproduction in any medium, provided the original author and source are credited.

\section{Introduction}

Autopsy, derived from the Greek 'autos' and 'opsomeri' means 'to see for oneself', originates from mummification using human dissection around 3000 BC. Over five millennia it has facilitated the study of human anatomy, physiology, and pathophysiology culminating in 21 st century medicine. Autopsy is divided into medicoscientific (consented, usually by a relative) and medico-legal (required by legal authority such as HM Coroner in the UK). For the last fifty years a worldwide decline in consented autopsy has occurred, such that in many countries it is on the brink of extinction [1]. For example, 30 years ago, consented autopsy rates were around $25 \%$ of inpatient deaths in the UK whereas in 2013 the average rate was less than $1 \%$. Despite the rapid decline, autopsy continues to benefit 21st century medicine, including roles in microbiology and diagnostics.

\section{Autopsy Microbiology}

Consented autopsy has two main microbiological roles in modern medicine. Firstly; to either confirm an antemortem infectious diagnosis or culture and identify undiagnosed antemortem pathogenic organisms. Infectious disease continues to feature as a major cause of mortality worldwide, especially in the developing world, where autopsy has been shown to identify a plethora of undiagnosed antemortem microbial pathologies [2].

There is, and has been for over a century, florid contention amongst the medical profession over the diagnostic use of post-mortem blood and tissue cultures [3-5]. The basis of the controversy stems from false positive results due to post-mortem indigenous bacterial invasion into the circulation and is founded in two main postulates: transmigration and agonal spread [5]. Transmigration was observed by Gradwohl, as bloodstream bacterial contamination due to the migration of mucosal or tissue flora occurring after the cessation of the systemic circulation leading to a rapidly deficient functional immune system [5,6]. Later, Fredette described agonal spread-bloodstream bacterial contamination during the agonal period due to a declining systemic circulation $[5,7]$.

In order to reduce false positive results, it is recommended that autopsy occurs aseptically, promptly and with preceding cadaveric refrigeration [5]. A key retrospective review of over 2,000 autopsies, published by Carpenter et al. showed that the rate of positive postmortem blood cultures were positively correlated with time after death $[5,8]$. However other similar studies have failed to reproduce this and argue against the importance of post-mortem bacterial transmigration [5-9]. Controversy remains.

The second major role of microbiology in autopsy is to enable the consented removal/examination of tissue and cultures of microorganisms to better our understanding of disease and its pathogenesis. There is a long and thriving history of autopsy providing a pivotal role in the advancement of medical knowledge. Welch et al. discovered a novel bacterium at autopsy in 1891 [10], and since autopsy studies have aided our understanding in invasive fungal disease [11], toxoplasma gondii [12], congenital rubella syndrome [13]. Severe acute respiratory syndrome (SARS) [14], tuberculosis/HIV coinfection [15] and cerebral malaria [16] to name but a few. Most recently consented fetal autopsy has aided in the mounting evidence of a causal link between Zika virus and microcephaly [17]. It is postulated that a lack of autopsy studies into middle east respiratory syndrome (MERS), a modern major public health epidemic with a case fatality of $30 \%$, is one of the reasons behind our reduced knowledge of this coronavirus compared to SARS [14].

\section{Autopsy Diagnostics}

A commonly held belief amongst physicians is that classical postmortem is outdated in the current era of sophisticated antemortem diagnostics $[18,19]$. There is a widespread belief that the opportunity for autopsy to reveal major discrepancies between antemortem and post-mortem diagnosis has long past [20,21]. One study found that $81 \%$ of clinicians believe advances in CT imaging have reduced the value of autopsy [18]. Conflicting research shows little change in misdiagnosis rates over recent decades [22-24] and that modern medicine continues to have significant misdiagnosis rates suggesting the role of autopsy as a useful determinant of underlying pathology remains [21,24-29].

Twenty-two percent of death certificates have been shown to have, "no value at all because of inadequate diagnosis" [30]. Death certificates are frequently completed by junior medical staff and their worryingly high innacuracy rates contribute to misleading mortality statistics. Autopsy acts as a gold standard for diagnostics which, if widely used, would result in highly accurate morbidity and mortality statistics and thus improved epidemiological data to base resource allocation.

Modern medicine means the population are living longer and accumulating co-morbidities and complex disease. Autopsy enables the interaction of diseases to be studied and hence aid in our understanding of their clinical picture. From comparing antemortem and postmortem diagnoses, clinicians can improve clinicopathological correlation, leading to improved antemortem diagnostics. This would lead to improved detection of disease enabling prompt treatment and also reduced false-positive diagnoses, hence reducing avoidable iatrogenic harm.

A large study of many thousands of autopsies in American intensive care units showed that $8 \%$ of patients died with a class 1 diagnostic error-had the diagnosis been made antemortem then the patient would 
have has an altered treatment plan and potential increased survival [28]. Intensive care patients are some of the most investigated patients in a hospital which begs the question-what proportion of general medical/surgical patients die misdiagnosed? The most prevalent misdiagnoses in the intensive care setting are pulmonary embolism, myocardial infarction, pneumonia and aspergillosis-all of which are commonly treatable if not preventable [28]. Furthermore, only $48 \%$ of patients who die due to myocardial infarction have the diagnosis on their death certificate-if we can increase our antemortem diagnosis then prompt treatment can be initiated and lives saved [30].

Importantly, autopsy should not be restricted to deaths that are complex or unexpected. Death in the terminally ill and elderly provide invaluable information on mortality, diagnostics, comorbidities, and training but also benefits the bereaved families.

Consented autopsy enables advances in medical care and diagnostics-resulting in safer care. For example, autopsy has been used in the development of gene therapies, medical procedures, surgical techniques, pharmacology, diagnostic tests and implantable devices [31-33]. The power of autopsy in the development of new technology is demonstrated historically by Rene Laennec (1781-1826) who invented the stethoscope. Laennac used his new instrument to listen to the sounds of the heart and lungs, but was only able to correlate these sounds to pathology via autopsy [34]. Consented autopsy will continue to inspire clinicians to develop new techniques to improve our quality of care.

\section{Combating the Decline}

The terminal decline of consented autopsy has shown few signs of slowing-primarily driven by a lack of clinician request for autopsy due to erroneous beliefs that autopsy is outdated and that consent is rarely granted. Combined with this is a general apathy towards autopsy amongst pathologists and ever increasing financial constraints. Despite this, there are many examples where simple interventions such as physician training and common place autopsy requests have resulted in rapid increases in consented autopsy rates $[35,36]$. These pilot studies prove that autopsy can be revived if the enthusiasm exists.

\section{Summary}

Autopsy has been used for millennia to advance our understanding of medicine and science. While controversies still exist over the use of microbiological diagnostic autopsy culture; there is still a strong and significant role for consented autopsy to enable the removal of tissue for research. Using autopsy appropriately, the link between clinical presentation, investigation results and diagnosis can be improvedindividuals can learn from previous mistakes and safety of care could improve. The evidence for consented autopsy is strong yet the decline in autopsy rates has unfortunately been stronger. It is time for the medical community to act, to revive enthusiasm and ensure that consented autopsy is appropriately offered, attended and learnt fromotherwise this invaluable diagnostic source will be extinct imminently throughout much of the world.

\section{References}

1. Turnbull A, Osborn M, Nicholas N (2015) Hospital autopsy: Endangered or extinct? J clin path 2014.

2. Martinez MJ, Massora S, Mandomando I (2016) Infectious cause of death determination using minimally invasive autopsies in developing countries. J diag microbio 84: 80-86.
3. Moulin GC, Love W (1988) The Value of Autopsy Microbiology. JCM 10: 21.

4. Niwayama G (1971) Postmortem Blood Microbiology Using Sterile Autopsy Technique. Tohoku J exp Med 105: 247-256.

5. Reidel S (2014) The Value of Postmortem Microbiology Cultures. J Clin Microbiol 52: 1028-1033.

6. Gradwohl RBH (1904) Importance de lexamen bacteriolique practique sur les cadavers. Ann Inst Pasteur (Paris) 18: 767-773.

7. Fredette JW (1916) Bacteremias in the agonal period. J Clin Med 2: 180-188.

8. Carpenter HM, Wilkins RM (1964) Autopsy bacteriology: review of 2,033 cases" Arch Pathol 77: 73-81.

9. Saegeman V, Verhaegen J, Lismont D, Verduyckt B, De Rijdt T, et al. (2009) Influence of postmortem time on the outcome of blood cultures among cadaveric tissue donors. Eur J Clin Microbiol Infect Dis 28:161-168.

10. Lucey BP, Hutchins GM (2004) "William H. Welch, MD, and the discovery of Bacillus welchii”. Arch Pathol Lab Med 128: 1193-1195.

11. Ruangritchankul K, Chindamporn A, Worasilchai N, Poumsuk U, Keelawat S, et al. (2015) Invasive fungal disease in university hospital: a PCR-based study of autopsy cases. Int J Clin Exp Pathol 8: 14840-14852.

12. Vijaykumar BR, Swathi UL (2016) Genetic characterization of Toxoplasma gondii from autopsy proven cases of AIDS associated cerebral toxoplasmosis in South India. Infect Genet Evol 39: 106-112.

13. Lazar M, Perelygina L, Martines R (2016) Immunolocalization and Distribution of Rubella Antigen in Fatal Congenital Rubella Syndrome. EBioMedicine 3: 86-92.

14. Zhou J, Chu H, Fuk-Woo Chan J, Yuen K (2015) Middle East respiratory syndrome coronavirus infection: virus-host cell interactions and implications on pathogenesis. Virology 12:218.

15. Bykhalov LS, Smimov AV (2015) Morphological characteristics of inflammation in HIV-associated pulmonary tuberculosis with regard to the expression of myeloperoxidase. Arkh Patol 77: 15-20.

16. Feintuch CM, Saidi A, Karl S, Chend G, Goldman-Yassen A, et al. (2016) Activated Neutrophils Are Associated with Pediatric Cerebral Malaria Vasculopathy in Malawian Children. MBio 7: e01300-01315.

17. Mlakar J, Korva M, Tul N, Popović M, Poljšak-Prijatelj M, et al. (2016) “Zika Virus Associated with Microcephaly". N Engl J Med 374:951-958.

18. Midelfart J, Aase S (1998) The value of autopsy from a clinical point of view: A survey of 250 general practitioners and hospital clinicians in the county of Sor-Trondelag, Norway. APMIS 106: 693-698.

19. Nemetz PN, Tanglos E, Sands LP, Fisher WP, Newman WP, et al. (2006) "Attitudes toward the autopsy--an 8-state survey." MedGenMed 8: 80.

20. Karwinski B, Hartveit F (1989) Death certification: increased clinical confidence in diagnosis and lack of interest in confirmation by necropsy is not justified. J ClinPathol 42: 13-17.

21. Shojania KG, Burton EC, McDonald KM, Goldman L (2003) "Changes in rates of autopsy-detected diagnostic errors over time: a systematic review." JAMA 289: 2849-2856.

22. Kirch W, Schafii C (1996) Misdiagnosis at a university hospital in 4 medical eras. Medicine (Baltimore) 75: 29-40.

23. Goldman L, Sayson R, Robbins S, Cohn LH, Bettmann M, et al. (1983) The value of the autopsy in three medical eras. N Engl J Med 308: 1000-1005.

24. Kirch W, Shapiro F, Folsch U (2004) Health care quality: Misdiagnosis at a university hospital in five medical eras. J Public Health 12: 154-161.

25. Bombí JA, Ramírez J, Solé M (2003) Clinical and Autopsy Correlation Evaluated in a University Hospital in Spain (1991-2000). 199: 9-14.

26. Twigg SJ, McCrirrick A, Sanderson PM (2001) A comparison of post mortem findings with post hoc estimated clinical diagnoses of patients who die in a United Kingdom intensive care unit. Intensive Care Med 27: 706-710.

27. Attems J, Arbes S, Bohm G, Böhmer F, Lintner F (2004) The clinical diagnostic accuracy rate regarding the immediate cause of death in a 
hospitalized geriatric population; an autopsy study of 1594 patients. Wien Med Wochenschr 154: 159-162.

28. Winters B, Custer J, Galvagno SM, Colantuoni E, Kapoor SG, et al. (2012) Diagnostic errors in the intensive care unit: a systematic review of autopsy studies. BMJ Qual Saf 21: 894-902.

29. Ornelas-Aguirre JM, Vazquez-Camacho G, Gonzalez-Lopez L (2003) Concordance between premortem and postmortem diagnosis in the autopsy: results of a 10-year study in a tertiary care center. Ann DiagnPathol 7: 223-230.

30. Ravakhah K (2006) Death certificates are not reliable: revivification of the autopsy. South Med J 99: 728-733.

31. Ayoub T, Chow J (2008) The conventional autopsy in modern medicine." J R Soc Med 101: 177-181.
32. Libow LS, Neufeld RR (2008) The autopsy and the elderly patient in the hospital and the nursing home: enhancing the quality of life. Geriatrics 63: 14-18.

33. Shojania KG, Burton EC (2008) The vanishing nonforensic autopsy. N Engl J Med 358: 873-875.

34. Roguin A (2006) Rene Theophile Hyacinthe Laennec (1791-1826): The Man Behind the Stethoscope. Clinical Medicine and Research 4:230-235.

35. Limacher E, Carr U, Bowker L, Ball RY (2007) Reversing the slow death of the clinical necropsy: developing the post of the Pathology Liaison Nurse. J Clin Pathol 60: 1129-1134.

36. Fowkes M (2016) Autopsies are vital to medicine. Don't let them go extinct. STAT. 